\title{
Case Report Vertebral Artery Thrombosis in Chronic Idiopathic Thrombocytopenic Purpura
}

\author{
Zakaria Hindi, ${ }^{1}$ Nirmal Onteddu, ${ }^{1}$ Christopher A. Ching, ${ }^{2}$ and Abdallah A. Khaled ${ }^{3}$ \\ ${ }^{1}$ Department of Internal Medicine, Texas Tech University Health Sciences Center at the Permian Basin, Odessa, TX, USA \\ ${ }^{2}$ School of Medicine, Texas Tech University Health Sciences Center at the Permian Basin, Odessa, TX, USA \\ ${ }^{3}$ School of Medicine, University of Jordan, Amman, Jordan \\ Correspondence should be addressed to Zakaria Hindi; dr.zhindi@hotmail.com
}

Received 27 February 2017; Revised 3 May 2017; Accepted 14 May 2017; Published 11 June 2017

Academic Editor: Massimo Gentile

Copyright (C) 2017 Zakaria Hindi et al. This is an open access article distributed under the Creative Commons Attribution License, which permits unrestricted use, distribution, and reproduction in any medium, provided the original work is properly cited.

\begin{abstract}
Introduction. Immune thrombocytopenic purpura (ITP) is an autoimmune hematological disorder that causes decreased production and destruction of platelets leading to thrombocytopenia. Although thrombocytopenia usually causes hemorrhagic problems, thrombotic events like strokes, although rare, can still occur. Management of thrombotic events in patients with ITP differs from that of patients with normal platelet count function and count. Case Description. A 32-year-old female with a history of ITP presented with ischemic stroke. The patient was treated in the hospital with IV immunoglobulin, discharged to a rehabilitation facility, and had complete resolution of symptoms when examined at a follow-up visit 3 months later. Conclusion. Although stroke in patients with ITP is very rare due to thrombocytopenia, it has been reported in several other published cases and is likely associated with increased platelet microparticle levels, a byproduct of platelet destruction. While usage of antiplatelet therapy in such patients is debated, immunosuppression therapy has been the mainstay treatment in all published cases.
\end{abstract}

\section{Introduction}

Idiopathic thrombocytopenia purpura is an autoimmune hematological disorder characterized by the destruction of the platelets, which is mediated through antibodies that bind to platelet specific antigens. The antibody-platelet antigen complex is subsequently removed in the spleen by macrophages. If untreated, the continued destruction and removal by spleen can lead to severe thrombocytopenia and severe bleeding. Although bleeding is the usual complication, thrombotic events including cerebrovascular accidents may rarely occur $[1,2]$. Thereby, we would like to report a case of female with chronic ITP who developed left vertebral artery thrombosis with left cerebellar infarction.

\section{Case Description}

A 32-year-old female with a history of chronic idiopathic thrombocytopenia purpura (ITP) presented to emergency room with vertigo and imbalance for two hours prior to admission. Physical examination was remarkable for cerebellar ataxia, positive Romberg's sign, dysmetria, and dyskinesia on the left upper limb. Urgent CT scan of head was done and showed no signs of intracranial hemorrhage. Complete blood count (CBC) on admission was only remarkable for platelet count of $49 \mathrm{~K} / \mathrm{uL}$. The rest of blood work-up including coagulation profile and comprehensive metabolic panel was unremarkable. The decision was made to start the patient on intravenous immunoglobulins (IVIG) while investigating for cerebrovascular accident. MRI for head and neck was done and showed infarction areas in left cerebellar hemisphere (Figure 1 (Radiology Department at Hamad General Hospital, Doha, Qatar)). MRA (Figure 2 (Radiology Department at Hamad General Hospital, Doha, Qatar)) for head and neck was done as well, which showed an occlusion in the distal segment of left vertebral artery. Transthoracic echocardiogram showed no thrombus formation in left atrium. Further investigations including factor $\mathrm{V}$ Leiden mutation, proteins $\mathrm{C}$ and $\mathrm{S}$ activity, 


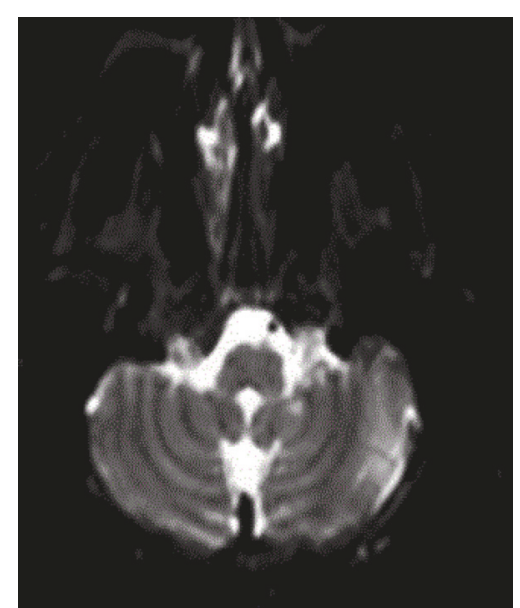

FIGURE 1: MRI of head on admission, showing multiple infarctions in the left cerebellum.

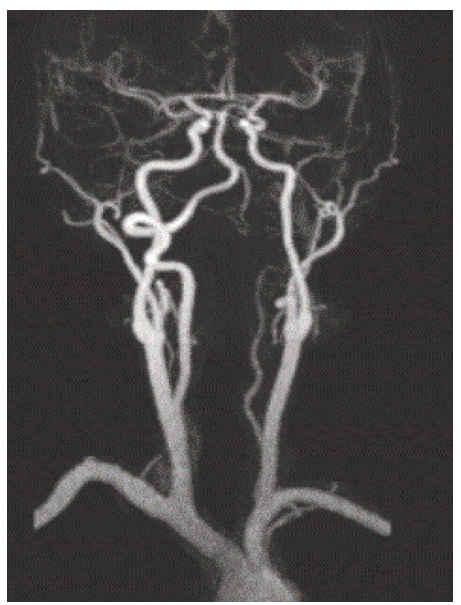

FIGURE 2: MRA of head and neck on admission, showing incomplete occlusion of left vertebral artery.

complement 3 and 4 levels, anti-phospholipid antibodies, anti-nuclear antibody, and antithrombin 3 level were all unremarkable. After consulting hematology and neurology team, the decision was to avoid both anticoagulant therapy for venous thromboembolism and antiplatelet therapy and to continue IVIG for five days total. One week later, the patient's symptoms improved with some residual difficulty walking. Average platelets count in the one-week period was $45 \mathrm{~K} / \mathrm{uL}$. The patient was transferred to a rehabilitation facility, where she continued to improve and was discharged afterwards. Three months later, in a follow-up visit, MRA for neck showed complete occlusion of left vertebral artery (Figure 3 (Radiology Department at Hamad General Hospital, Doha, Qatar)). MRI for head showed complete resolution of the cerebellar infarction (Figure 4 (Radiology Department at Hamad General Hospital, Doha, Qatar)). CBC was obtained during the visit and her platelet count was $46 \mathrm{~K} / \mathrm{uL}$. The patient was completely asymptomatic and was advised to avoid antiplatelet therapy.

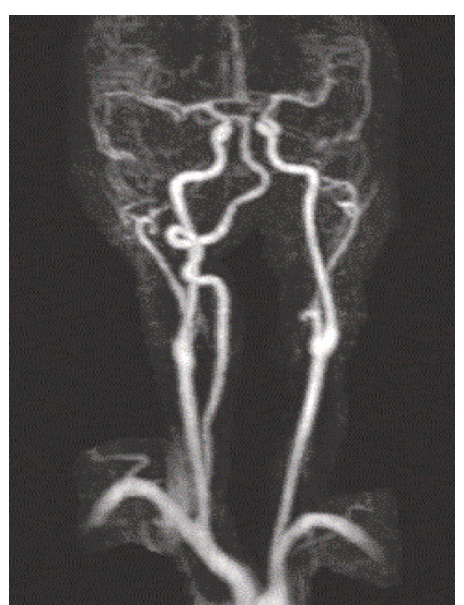

FIGURE 3: MRA of head and neck at 3-month follow-up visit.

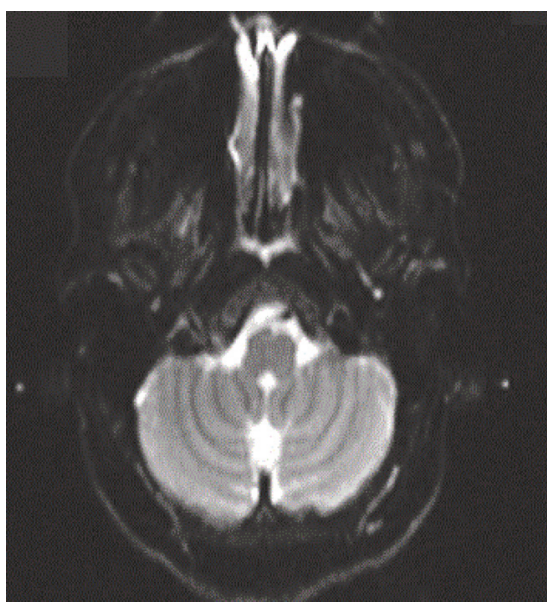

FIGURE 4: MRI of head and neck at 3-month follow-up visit.

\section{Discussion}

Eight published cases of ITP with ischemic stroke were found after reviewing the literature, none of which described vertebral artery thrombosis. The sites of the infarction in seven of the cases were in cortical regions [3-9] and only one with thalamic infarction [10]. Out of the reports with cortical infarctions, only two were involving the posterior circulation (occipital lobes) $[6,9]$. The etiology of ischemia in these cases seemed to be thrombotic rather than embolic, as there were no risk factors that favored embolic events (Table 1 summarizes the main characteristics of ITP patients [3-10]).

The association between thrombosis and ITP is still not well established. However, evidence has shown that platelet microparticles (PMP) have a major role in thrombogenesis in ITP. PMP is a substance that is produced from platelets upon destruction. Studies have shown that the levels of PMP are higher in both ITP and non-ITP patients with cerebral ischemic changes compared to healthy individuals. Furthermore, the high level PMP was found to be protective against hemorrhage. However, in excess levels, PMP can 
TABLE 1: Summary of published cases (ITP: idiopathic thrombocytopenia purpura, HTN: hypertension, and APS: antiphospholipid syndrome).

\begin{tabular}{lcccc}
\hline $\begin{array}{l}\text { Author of } \\
\text { reported cases }\end{array}$ & Age of the patient & Gender & Site of infarction & Risk factors \\
\hline $\begin{array}{l}\text { Otsuki et al. } \\
\text { Gümüş and }\end{array}$ & 38 & Female & Left temporal and parietal regions & APS and ITP \\
Yilmaz & 55 & Female & Left middle cerebral artery territory & ITP \\
Hashmi & 57 & Male & Right middle cerebral artery territory & ITP \\
Theeler and Ney & 63 & Male & Right occipital lobe infarction & ITP \\
Peña et al. & 84 & Male & Left thalamic infarction & ITP \\
Ichijo et al. & 60 & Female & Middle cerebral artery territory & ITP \\
Mahawish et al. & 79 & Male & Multiple focal ischemic lesions in both cerebral hemispheres & HTN and ITP \\
Nanri et al. & 31 & Male & Right occipital lobe and hippocampal gyrus & Migraine headaches and ITP \\
\hline
\end{tabular}

promote thrombin formation and thus thrombosis [11-14]. Other mechanisms of thrombogenesis in ITP are the treatment with IVIG. IVIG can promote thrombosis by increased blood viscosity and increased thrombin production and by directly affecting vascular endothelium with associated cerebral arterial vasospasm [15-18].

The treatment of ITP ischemic stroke is controversial as both hematologist and neurologist have different perspectives. All mentioned cases have used immunosuppression therapy (IVIG, steroids, or cyclosporine). The importance of immunosuppression in the setting of ITP and ischemic stroke likely is due to the resulting decrease of PMP levels, as mentioned by Ichijo et al. [7]. As for antiplatelet therapy, the decision varied among the cases. In two of them, antiplatelet therapy was given and there were no hemorrhagic events $[4,7]$. On the other hand, antiplatelet therapy was withheld in three cases without any further adverse thrombotic events $[5,8,10]$. Other treatments such as calcium channel blockers were given in two cases. Since platelet fragmentation is calcium dependent, these agents can prevent the release of PMP by inhibiting extracellular calcium influx $[13,14]$.

\section{Conclusion}

Treatment of ischemic stroke in ITP is still controversial; nevertheless, the use of immunosuppression modality and consulting both hematology and neurology teams are essential for initial plan of care.

\section{Conflicts of Interest}

The authors have no conflicts of interest.

\section{References}

[1] R. McMillan, "The pathogenesis of chronic immune (idiopathic) thrombocytopenic purpura," Seminars in Hematology, vol. 37, no. 1, pp. 5-9, 2000.

[2] H.-K. Park and S.-H. Lee, "Ischemic stroke associated with immune thrombocytopenia: lesion patterns and characteristics," Neurological Sciences, vol. 35, no. 11, pp. 1801-1806, 2014.
[3] T. Otsuki, T. Funakawa, T. Sugihara et al., "Multiple cerebral infarctions in a patient with refractory idiopathic thrombocytopenic purpura," Journal of Internal Medicine, vol. 241, no. 3, pp. 253-256, 1997.

[4] H. Gümüş and H. Yılmaz, "Severe ischemic stroke in a patients with idiopathic thrombocytopenic purpura: a case report," Turkiye Fiziksel Tip ve Rehabilitasyon Dergisi, vol. 61, no. 2, pp. 171-174, 2015.

[5] I. Hashmi, "Ischaemic stroke in a patient with refractory idiopathic thrombocytopenic purpura: an unusual clinical dilemma," Journal of Medical Cases, vol. 3, no. 3, pp. 204-206, 2012.

[6] B. J. Theeler and J. P. Ney, "A Patient with Idiopathic Thrombocytopenic Purpura Presenting with an Acute Ischemic Stroke," Journal of Stroke and Cerebrovascular Diseases, vol. 17, no. 4, pp. 244-245, 2008.

[7] M. Ichijo, S. Ishibashi, T. Ohkubo et al., "Elevated platelet microparticle levels after acute ischemic stroke with concurrent idiopathic thrombocytopenic purpura," Journal of Stroke and Cerebrovascular Diseases, vol. 23, no. 3, pp. 587-589, 2014.

[8] K. Mahawish, N. Pocock, S. Mangarai, and A. Sharma, "Cerebral infarction in idiopathic thrombocytopenic purpura: a case report," BMJ Case Reports, vol. 2009, Article ID bcr04.2009.1748, 2009.

[9] K. Nanri, K. Niiyama, H. Utsumi et al., "A case of migrainous infarction accompanying idiopathic thrombocytopenic purpura," Rinsho shinkeigaku, vol. 42, no. 9, pp. 868-872, 2002.

[10] A. D. L. Peña, J. Fareed, I. Thethi, S. Morales-Vidal, M. J. Schneck, and D. Shafer, "Ischemic stroke in the setting of chronic immune thrombocytopenia in an elderly patient-a therapeutic dilemma," Clinical and Applied Thrombosis/Hemostasis, vol. 18, no. 3, pp. 324-326, 2012.

[11] R. Flaumenhaft, "Formation and fate of platelet microparticles," Blood Cells, Molecules, and Diseases, vol. 36, no. 2, pp. 182-187, 2006.

[12] P. J. Sims, E. M. Faioni, T. Wiedmer, and S. J. Shattil, “Complement proteins C5b-9 cause release of membrane vesicles from the platelet surface that are enriched in the membrane receptor for coagulation factor Va and express prothrombinase activity," Journal of Biological Chemistry, vol. 263, no. 34, pp. 18205-18212, 1988.

[13] Y. J. Lee, W. Jy, L. L. Horstman et al., "Elevated platelet microparticles in transient ischemic attacks, lacunar infarcts, and 
multiinfarct dementias," Thrombosis Research, vol. 72, no. 4, pp. 295-304, 1993.

[14] Y. S. Ahn, L. L. Horstman, W. Jy, J. J. Jimenez, and B. Bowen, "Vascular dementia in patients with immune thrombocytopenic purpura," Thrombosis Research, vol. 107, no. 6, pp. 337344, 2002.

[15] W. H. Reinhart and P. E. Berchtold, "Effect of high-dose intravenous immunoglobulin therapy on blood rheology," The Lancet, vol. 339, no. 8794, pp. 662-664, 1992.

[16] M. C. Dalakas, "High-dose intravenous immunoglobulin and serum viscosity: risk of precipitating thromboembolic events," Neurology, vol. 44, no. 2, pp. 223-226, 1994.

[17] A. S. Wolberg, R. H. Kon, D. M. Monroe, and M. Hoffman, "Coagulation factor XI is a contaminant in intravenous immunoglobulin preparations," American Journal of Hematology, vol. 65, no. 1, pp. 30-34, 2000.

[18] R. Voltz, F. V. Rosen, T. Yousry, J. Beck, and R. Hohlfeld, "Reversible encephalopathy with cerebral vasospasm in a Guillain-Barré syndrome patient treated with intravenous immunoglobulin," Neurology, vol. 46, no. 1, pp. 250-251, 1996. 


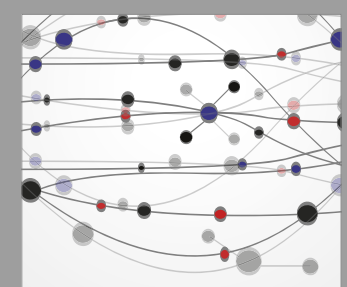

The Scientific World Journal
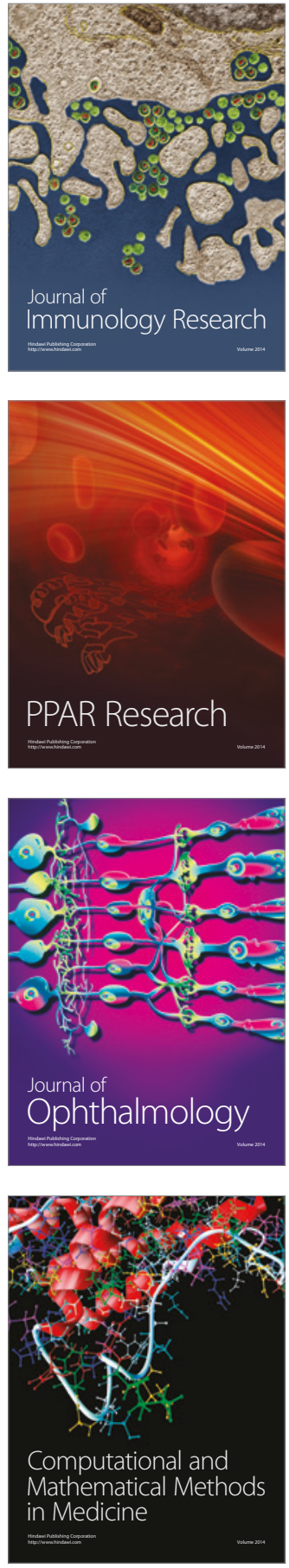

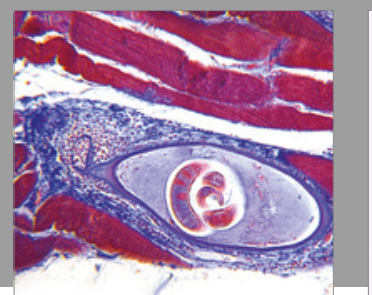

Gastroenterology Research and Practice
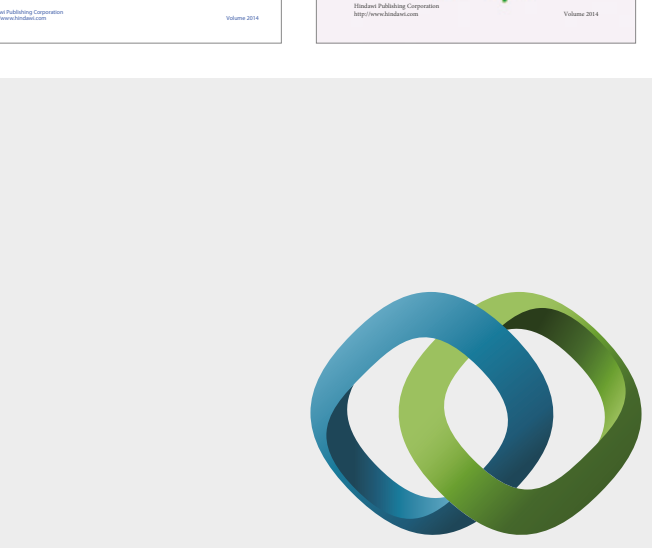

\section{Hindawi}

Submit your manuscripts at

https://www.hindawi.com
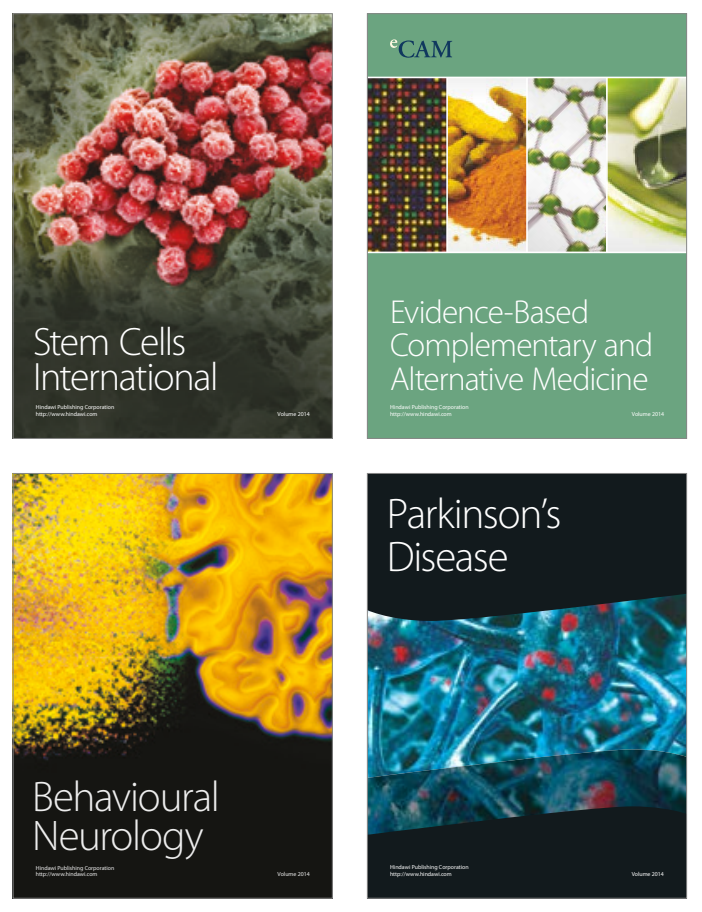
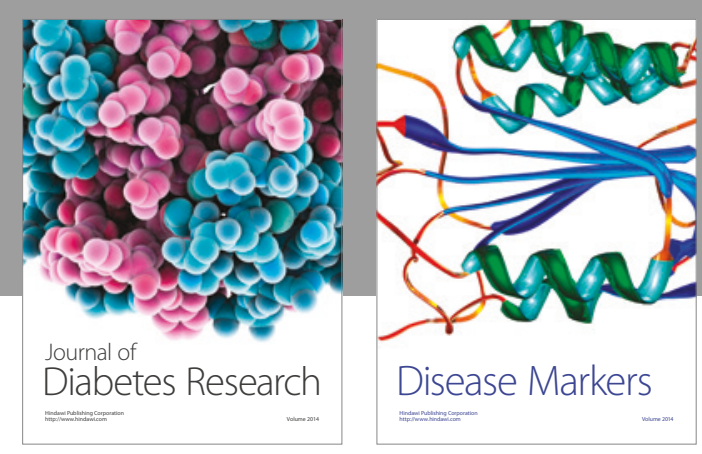

Disease Markers
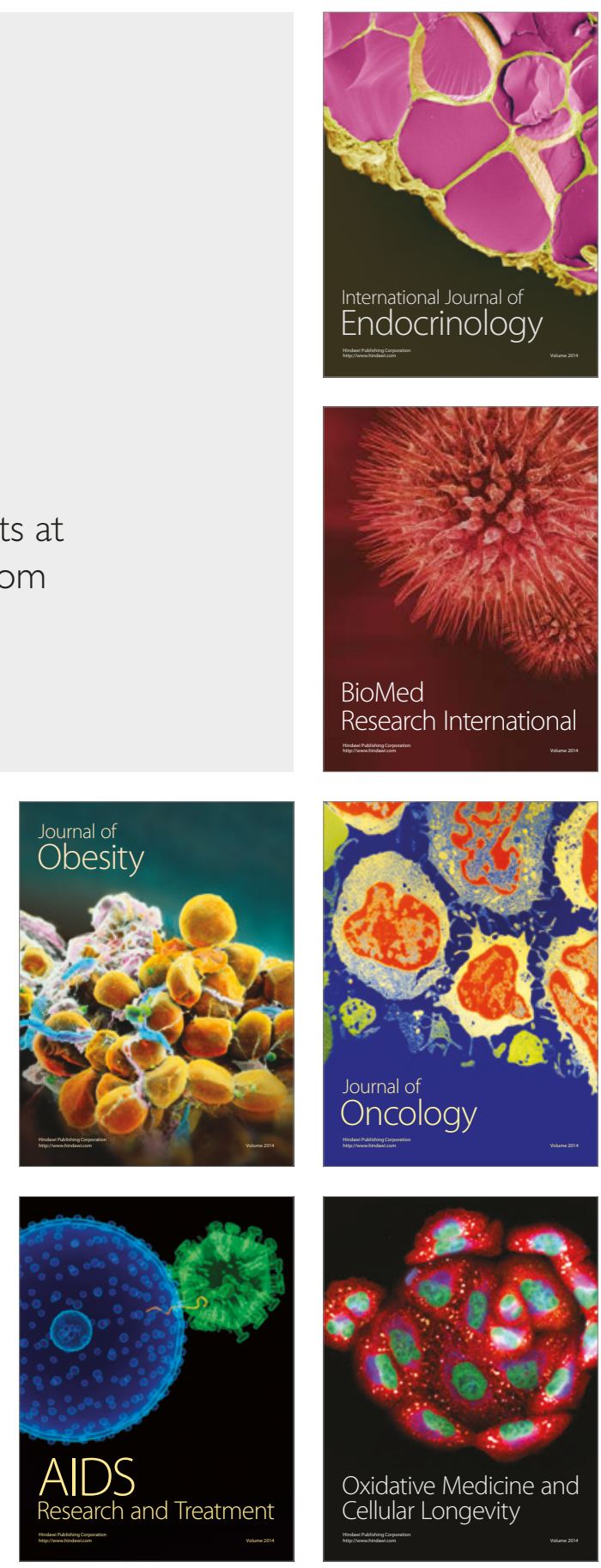